\title{
Epsilon near zero metamaterials for ultra-low power nonlinear applications
}

\author{
Monika Pietrzyk $^{a}$, Rashid Kaipurath ${ }^{b}$, Daniele Faccio $^{b}$ and Andrea Di Falco ${ }^{a}$ \\ ${ }^{a}$ School of Physics and Astronomy, University of St. Andrews, St. Andrews KY16 9SS, UK; \\ ${ }^{b}$ School of Eng. and Physical Sciences, Heriot-Watt University, Edinburgh EH14 4AS, UK
}

\begin{abstract}
Epsilon-near-zero metamaterial samples, composed of five alternating bi-layers of silica and silver, are fabricated using the electron-beam evaporator. Nonlinear properties of samples are measured using a pulsed Ti:sapphire laser by the z-scan technique. It is observed that the real part of the nonlinear Kerr index is one order of magnitude higher than the values expected from a naive averaging of the corresponding coefficients of metal and dielectric layers (the correct averaging should be performed with respect to the nonlinear susceptibility), so that its value is actually of the same order of magnitude as that of a single silver layer. At the same time, the transmission of our samples is remarkably higher than that of a single silver layer of the same thickness. These characteristics have a great impact on the amount of optical energy which can be pumped into the structure, thus allowing its nonlinear properties to be accumulated over long propagation distance along the sample. This property is very promising for applications, which are based on the modulation of phase, amplitude or frequency of light, especially those which require low-power operations, such as all-optical switching and memory elements.
\end{abstract}

Keywords: Epsilon-near-zero metamaterials, multilayer structures, effective medium theory, Kerr nonlinearity

\section{INTRODUCTION}

Epsilon-near-zero (ENZ) metamaterials are arrangements of artificially structured elements with dimensions much smaller than the wavelength of light, with a vanishing real part of the homogenised dielectric permittivity, for a given wavelength. Such structures can have a variety of applications, including tunnelling of electromagnetic energy through subwavelength channels ${ }^{1}$ cloaking $^{2}$ and subwavelength imaging. ${ }^{3}$ It has been also predicted that ENZ metamaterial structures can be used to boost Kerr nonlinearities. ${ }^{4,5}$

In this work we fabricate and experimentally investigate ENZ metamaterials composed of metal-dielectric multilayers. We observe ultrafast nonlinear response of our structures at the red edge of the visible spectrum. The effective nonlinearity turns out to be one order of magnitude higher than that of the constituent materials of the equivalent thickness. At the same time the observed absorption losses are about one order of magnitude lower than absorptions of a silver layer of the same thickness as our sample. In this way we overcome the fundamental limitations related to the relatively low nonlinear response of natural materials. This is an extremely desirable property for many applications in fundamental studies ranging from ultrashort pulse generation and compression, higher harmonic generation and quantum optics, to more practical applications, such as all-optical switching and memory elements and other ultra-low power optical systems.

\section{LINEAR PROPERTIES}

We fabricated ENZ metamaterial samples composed of five alternating bilayers, as sketched in fig. 1: dielectric (70 nm thick SiO2) layers with positive real part of the permittivity and metal (5 nm thick Ag) layers with the negative real part of the permittivity, deposited on a $1 \mathrm{~mm}$ thick borosilicate glass substrate. We used the the Edward auto 306 electron beam evaporator with a base pressure below $3 \times 10^{-6}$ mbar. To grant uniformity of the samples, the deposition rate was kept below $0.1 \mathrm{~nm} / \mathrm{s}$ for metals and below $0.3 \mathrm{~nm} / \mathrm{s}$ for silica. Smooth and continuous layers of silver, below the standard percolation limit, were obtained by seeding each metal layer with

Further author information: (Send correspondence to A.D.F., E-mail: adf10@st-andrews.ac.uk and D.F., E-mail: d.faccio@hw.ac.uk)

Photonic and Phononic Properties of Engineered Nanostructures V, edited by Ali Adibi,

Shawn-Yu Lin, Axel Scherer, Proc. of SPIE Vol. 9371, 93710Y · (c) 2015 SPIE

CCC code: $0277-786 \mathrm{X} / 15 / \$ 18 \cdot$ doi: $10.1117 / 12.2078884$

Proc. of SPIE Vol. $937193710 Y-1$ 


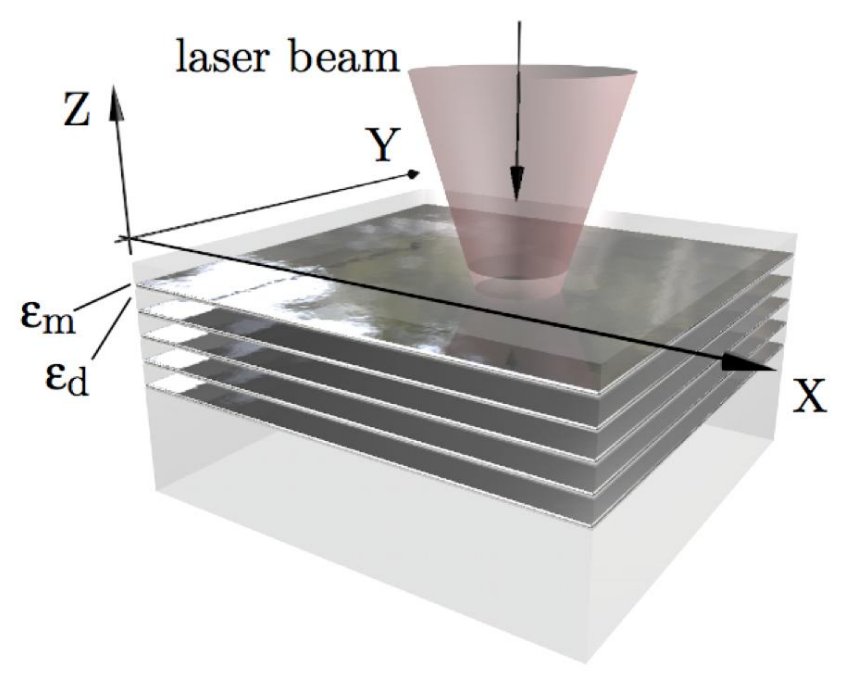

Figure 1. Sketch of the fabricated ENZ metamaterials.

$0.7 \mathrm{~nm}$ thick layer of germanium. ${ }^{7}$ The thicknesses of metal and dielectric layers has been optimised for the ENZ condition at the wavelength of $880 \mathrm{~nm}$. The development process was complemented by a combination of SEM, STEM and AFM measurements of the samples. The samples showed a high degree of uniformity, well below what measurable with the optical setup.

The linear properties of the ENZ metamaterial were measured by a standard setup composed of a collimated beam from an Ocean Optics HL2000 Halogen Lamp and two spectrum analysers. Under normal incidence illumination, the reflected and transmission signals for normal were collected and then used to retrieve the effective complex permittivity (the real, $\varepsilon_{r}$, and imaginary, $\varepsilon_{i}$, parts) of the multilayer samples by means of a standard least mean square fitting procedure. Those values were also compared with the theoretical values of the transmission and reflection coefficients, calculated using the transfer matrix method ${ }^{6}$ and a good agreement between them has been observed.

The results are shown in Fig. 2 in thick solid lines. The ENZ condition, $\varepsilon_{r}=0$, is satisfied for the wavelength $\lambda=880 \mathrm{~nm}$. To check that the metamaterials exhibit indeed a homogenised response, we compared the retrieved permittivity with the prediction of the effective theory. According to this approximation, for polarization direction transverse to the multilayer, the effective permittivity is given by $\varepsilon_{\mathrm{eff}}=\left(l_{\mathrm{m}} \varepsilon_{\mathrm{m}}+l_{\mathrm{d}} \varepsilon_{\mathrm{d}}\right) /\left(l_{\mathrm{m}}+l_{\mathrm{d}}\right)$, where $l_{\mathrm{m}}$ is the thickness of the metal layer, $l_{\mathrm{d}}$ is the thickness of the dielectric layer and $\varepsilon_{m}$ and $\varepsilon_{d}$ are the complex permittivities of the silver and silica respectively. These parameters were obtained by the explained retrieval procedure, applied to single layers of $\mathrm{SiO}_{2}$ and $\mathrm{Ge} / \mathrm{Ag}$ layers, specifically fabricated for the purpose. This approach also solves the issue of having to account for the complex metallic permittivity from first principle. In other words, our measurements use directly the actual metallic dispersion of the seeded silver layer, rather than an approximation. The results leave no doubts about the validity of the method. The dashed lines of Fig. 2 show the effective real and imaginary part of the homogenised permittivity. In particular the data show a remarkable agreement of the two approaches until well below a wavelength of $800 \mathrm{~nm}$. For shorter wavelengths the homogenisation approximation describes less and less accurately the multilayer. This happens when the constituent dielectric and metallic layers are roughly 10 times smaller and 150 smaller than the wavelength, respectively.

\section{NONLINEAR PROPERTIES}

To determine the effective behaviour of nonlinearity it is useful to work with the complex nonlinear Kerr index, $n_{2}=\left[3 /\left(4 \varepsilon_{0} c\right)\right] \chi^{(3)} /\left(n_{r}\left(n_{r}+i n_{i}\right)\right)$, where $\epsilon_{0}$ is the vacuum permittivity, $c$ is the speed of light in vacuum and $n_{r, i}$ are the real and imaginary part of the linear refractive index. The real and imaginary part of the Kerr index 


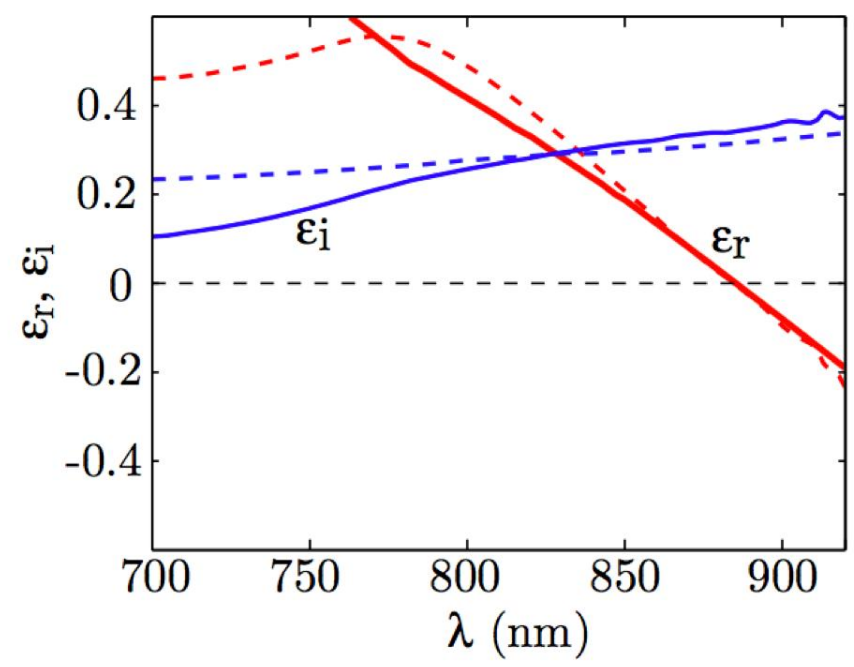

Figure 2. Measured real ( $\varepsilon_{r}$, red dashed curve) and imaginary ( $\varepsilon_{i}$, blue dashed curve) parts of the linear dielectric permittivity of the sample. The solid curves show the effective permittivity obtained started from the measurements of the single dielectric and metallic layers.

can be easily calculated, ${ }^{8} n_{2 r}=\frac{3}{4 \varepsilon_{0} c} \frac{n_{r} \chi_{r}^{(3)}+n_{i} \chi_{i}^{(3)}}{D}$ and $n_{2 i}=\frac{3}{4 \varepsilon_{0} c} \frac{n_{r} \chi_{i}^{(3)}-n_{i} \chi_{r}^{(3)}}{D}$, where $D=n_{r}\left(n_{r}^{2}+n_{i}^{2}\right)$, $n_{2 i}$ corresponds to the nonlinear absorption coefficient, $\beta_{2}=4 \pi n_{2 i} / \lambda$, and $\lambda$ is the vacuum wavelength. It is obvious that the parameters $n_{2 r}$ and $\beta_{2}$ depend, respectively, on sums and differences of the real and imaginary part of the linear refractive index, $n$, and also on the nonlinear susceptibility term, $\chi^{(3)}$. Therefore, the interplay between the real and imaginary part of the linear/nonlinear coefficient can be used to enhance or tailor the effective $n_{2 r}$ and $\beta_{2}$ coefficient. The fundamental role in determining the nonlinear behaviour close to the ENZ wavelength is played by the linear refractive index coefficients. By assuming $\chi_{r}^{(3)} \sim \chi_{i}^{(3)}$, which is verified in our experiments, the wavelength dependence of $n_{2 r}$ is basically determined by the dispersion of the linear refractive indices, i.e. the term $\left(n_{r}+n_{i}\right) / D$. Similarly, the nonlinear absorption coefficient is determined by the $\left(n_{r}-n_{i}\right) / D$ term. The presence of an ENZ wavelength significantly modifies the behaviour of the metamaterial. Namely, a peak is observed in the $\left(n_{r}+n_{i}\right) / D$ term, thus indicating a clear enhancement of $n_{2 r}$, while the nonlinear absorption shows a more complex behaviour and even changes the sign, being driven by the $\left(n_{r}-n_{i}\right) / D$ term. The transition from positive to negative nonlinear absorption passes through the zero nonlinear absorption at the ENZ wavelength, so that nonlinear phase shifts can be attained with minimum or zero nonlinear losses. This is a unique feature of the ENZ metamaterials, which underlines the crucial role played by the material linear dispersion and its effects on the nonlinear response of the metamaterial.

Measurements of our ENZ metamaterial samples were performed using a standard Z-scan technique. ${ }^{9}$ The laser source was a $100 \mathrm{fs}$, Ti:Sapphire source pumping a tunable wavelength optical parametric amplifier operating at the wavelengths ranging from 700 to $950 \mathrm{~nm}$. The measured nonlinear coefficients $n_{2 r}$ and $\beta_{2}$ are shown in fig. 3 and fig. 4 . The experimental data is represented by the circle points, each point has an error bar of approximately $\pm 10 \%$ of the measured values. The material clearly shows a large enhancement of the nonlinear Kerr index close to the ENZ wavelength. However, differently from other nonlinearity enhancement mechanisms based e.g. on plasmonic ${ }^{11}$ or microcavity resonances, ${ }^{10}$ this response has a very wide bandwidth and a relatively weak dependence on wavelength. Moreover, what also important, the nonlinear absorption coefficient drops to zero close to the ENZ wavelength and changes the sign for longer wavelengths.

The solid lines in fig. 3 and fig. 4 correspond to the theoretical predictions based on the effective medium theory, where the nonlinear parameters of silver are used as free fitting parameters, with the optimal values $n_{2 r}^{A g}=2.410^{-10} \mathrm{~cm}^{2} / \mathrm{W}$ and $\beta_{2}^{A g}=-2.410^{-5} \mathrm{~cm} / \mathrm{W}$, that agrees with the coefficients reported in the literature. ${ }^{12}$ The effective medium approach does qualitatively reproduce the experimental results and also captures the rather 


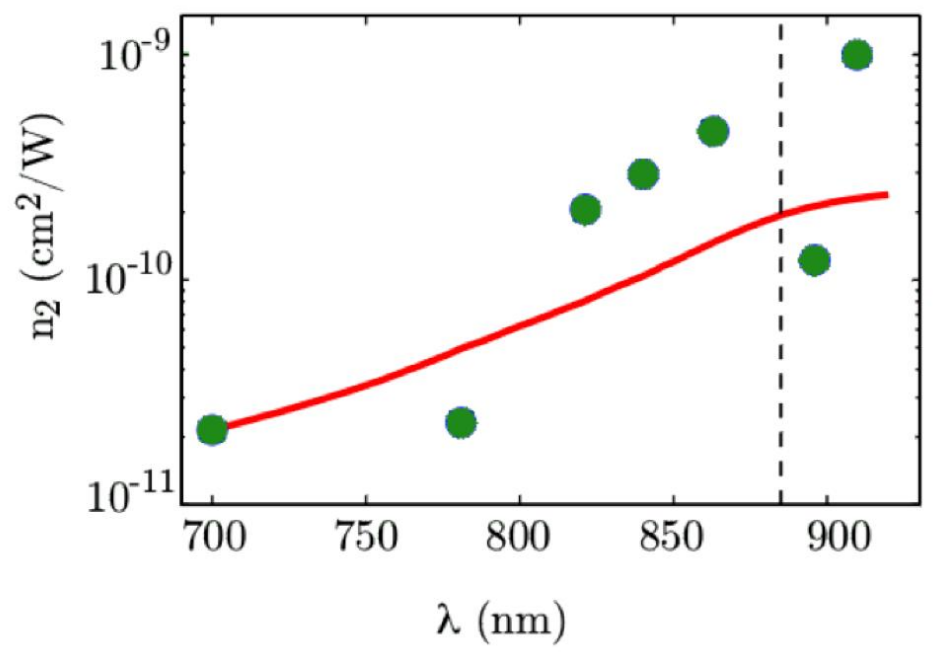

Figure 3. Experimental results (green circles) for the real part of the nonlinear Kerr index, around the ENZ wavelength indicated with a vertical dashed line. The solid red lines show the theoretical predictions based on an effective-medium theory.

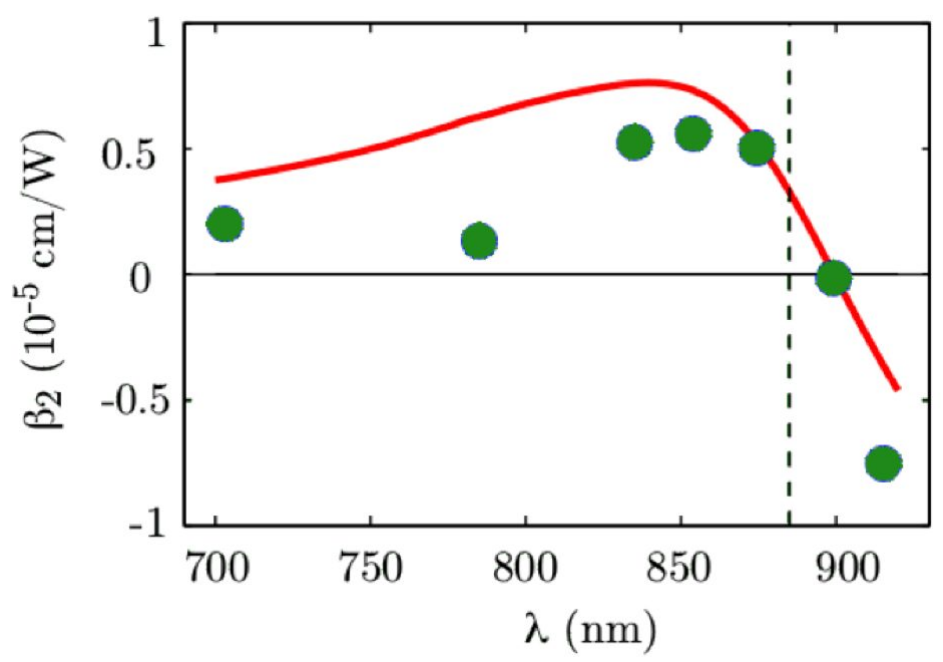

Figure 4. Experimental results (green circles) for the imaginary part of the nonlinear Kerr index, around the ENZ wavelength indicated with a vertical dashed line. The solid red lines show the theoretical predictions based on an effective-medium theory. 
unexpected inversion of the sign of $\beta_{2}$ close to the ENZ wavelength. This behaviour results from the non-trivial dependence of the overall nonlinear Kerr index, $n_{2}$, on both the real and imaginary part of the nonlinear susceptiibility $\chi^{(3)}$. The measured $n_{2 r}$ is so large that for the laser pulse intensities used in our experiments, the effective change of the nonlinear Kerr index, $\delta n=n_{2 r} I$, is of the same order of magnitude as real part of the linear refractive index, $n_{r}$. Taking into account also the fact that transmittivity of our sample with the total thickness of $380 \mathrm{~nm}$ is relatively high, $T \sim 10 \%$, this paves the way for various applications, such as optical switching, nonlinear frequency conversion through self-phase or cross-phase modulation and extreme nonlinear optics in thin films. ${ }^{4}$

\section{CONCLUSIONS}

We have shown that ENZ metamaterials have interesting properties from the point of view of applications in nonlinear optics, due to their ability to tailor and access novel nonlinear propagation regimes. The interplay between the linear and nonlinear, real and imaginary parts of the polarisation of the medium makes it possible to design metamaterials that exhibit nonlinearities with values similar to those of metals, while having substantially reduced losses. The giant phase shift in the ENZ multilayer structures is not achievable in the constituent materials alone. This leads to a novel and interesting behaviour, such as the observed change in the sign of $\beta_{2}$, which effectively eliminates nonlinear absorption close to the ENZ point. Moreover, the possibility to optically control the refractive index of the metamaterial by the same order of magnitude as the value of refractive index itself can be used to effectively tailor the impedance of the metamaterial and match it to that of the surrounding medium, thus making possible new forms of efficient optical switches and providing new platforms for non-perturbative nonlinear optics at low light intensities.

\section{Acknowledgements}

This work was supported by the EPSRC grant EP/ J004200/1. A.D. F. is a EPSRC career acceleration fellow (EP/I004602/1). D.F. acknowledges financial support from the European Research Council under the European Union Seventh Framework Programme (FP/2007-2013)/ERC GA 306559 and EPSRC (UK, Grant No. EP/J00443X/1). L.C. and M.C. acknowledge the support from the People Programme (Marie Curie Actions) of the European Union's FP7 Programme THREEPLE (GA 627478) and KOHERENT (GA 299522), respectively.

\section{REFERENCES}

[1] D. A. Powell, A. Alu, B. Edwards, A. Vakil, Y. S. Kivshar and N. Engheta, "Nonlinear control of tunnelling through an epsilon-near-zero channel," Phys. Rev. B 79, 245135-1-5 (2009).

[2] J. Hao, W. Yan and M. Qiu, "Super-reflection and cloaking based on zero index metamaterial," Appl. Phys. Lett. 96, 101109-1-3 (2010).

[3] G. Castaldi, S. Savoia, V. Galdi, A. Alu and N. Engheta, "Analytical study of subwavelength imaging by uniaxial epsilon-near-zero metamaterial slabs," Phys. Rev. B 86, 115123-1-10 (2012).

[4] A. Ciattoni, C. Rizza and E. Palange, "Extreme nonlinear electrodynamics in metamaterials with very small linear dielectric permittivity," Phys. Rev. A 81, 043839-1-12 (2010).

[5] C. Rizza, A. Di Falco and A. Ciattoni, "Gain assisted nanocomposite multilayers with near zero permittivity modulus at visible frequencies," App. Phys. Lett. 99, 221107-1-3 (2011).

[6] Z. Knittl, "Optics of Thin Films: An Optical Multilayer Theory," Wiley, London, (1976).

[7] W. Chen, M. D. Thoreson, S. Ishii, A. V. Kildishev and V. M. Shalaev, "Ultra-thin ultra-smooth and low-loss silver films on a germanium wetting layer," Opt. Exp. 18, 5124-5134 (2010).

[8] D. D. Smith, Y. Yoon, R. W. Boyd, J. Campbell, L.A. Baker, R. M. Crooks and M. George, "Z-scan measurement of the nonlinear absorption of a thin gold film," J. Appl. Phys. 86, 6200-6205 (1999).

[9] M. Sheik-Bahae, A. Said, T. -H Wei, D. Hagan and E. Van Stryland, "Sensitive measurement of optical nonlinearities using a single beam," IEEE J. Quant. Electron. 26, 760-769 (2007).

[10] J. B. Khurgin and G. Sun, "Third-order nonlinear plasmonic materials: Enhancement and limitations," Phys. Rev. A 88, 053838-1-14 (2013). 
[11] P. Genevet, J. -P. Tetienne, E. Gatzogiannis, R. Blanchard, M. A. Kats, M. O. Scully and F. Capasso, "Large Enhancement of Nonlinear Optical Phenomena by Plasmonic Nanocavity Gratings," Nano Lett. 10, 4880-4883 (2010).

[12] G. Ma and S. H. Tang, "Ultrafast optical nonlinearity enhancement in metallodielectric multilayer stacks," Opt. Lett. 32, 3435-3437 (2007).

Proc. of SPIE Vol. $937193710 Y-6$ 